\title{
On predicting the future states of awareness for recognition of unrecallable items
}

\author{
JASON L. HICKS \\ Louisiana State University, Baton Rouge, Louisiana \\ and \\ RICHARD L. MARSH \\ University of Georgia, Athens, Georgia
}

\begin{abstract}
Two experiments were conducted that examined people's predictive accuracy for their future states of awareness. In both experiments, participants learned word pairs and were tested with a cued recall test. When recall failed, predictive judgments were obtained. In Experiment 1, feeling of knowing judgments predicted not only future recognition but also the state of awareness as remembered or known that was associated with that recognition. In Experiment 2, predictive remember-know judgments were found to be accurately diagnostic of future states of awareness as well. One conclusion to be drawn from these data is that recollective details are among the partial knowledge that is retrieved in the absence of successful cued recall. General implications for theories of feeling of knowing are discussed.
\end{abstract}

In a now-classic article, Tulving and Pearlstone (1966) demonstrated that cued recall performance was generally better than free recall performance. Their article made the important point that items that are not accessible to free recall processes can nevertheless have sufficient availability to be recalled in the presence of the correct memorial cues. In the limit case, when an item is presented intact on a recognition test (i.e., a copy cue), performance is almost always better than when a person is tested with free recall (but see Tulving \& Thomson, 1973).

When an item cannot be recalled, many researchers have been interested in investigating the properties of the memorial information that is nevertheless available for that item. For example, Brown and McNeill (1966) introduced a paradigm for studying tip-of-the-tongue(TOT) states. People in a TOT state can correctly produce information about the first letter of the word that they are attempting to retrieve, as well as the number of syllables (e.g., Koriat, 1993; Koriat \& Lieblich, 1974). Such results suggest that when recall fails, people are able to retrieve partial information about the items for which they are searching. Although TOT research often uses general knowledge questions, investigations of feeling of knowing (FOK) often use paired associates to make the same general claim concerning the retrieval of partial episodic information. In Hart's (1965) seminal work on FOK, people demonstrated that when they could not recall the target in the presence of the cue for a partic-

We thank Joshua Reese for his dedicated help in collecting the data. Correspondence should be addressed to R. L. Marsh, Department of Psychology, University of Georgia, Athens, GA 30602-3013 (e-mail: jhicks@1su.edu or rlmarsh@uga.edu).

-Accepted by previous editorial team ular word pair, they could accurately predict whether they would be able to recognize the target from among several distractors. In fact, generalizations are often drawn between FOK and TOT experiments (e.g., Yaniv \& Meyer, 1987) because variables frequently affect these judgments in similar ways and because the two judgments appear to be correlated with each other (e.g., Metcalfe, Schwartz, \& Joaquim, 1993).

In the present study, our goal was to determine whether the information that is available when recall fails is predictive of later recognition performance (as has been shown in the FOK literature), but in addition, whether it would be predictive of the state of awareness associated with recognition. Our reasoning was as follows: Memory traces are often depicted as bundles of features (e.g., Anisfield \& Knapp, 1968; Bower, 1967; Dewhurst \& Conway, 1994; Johnson, Hashtroudi, \& Lindsay, 1993). When these features are not recorded in sufficient detail or number to support recall, their partial retrieval has been shown in the FOK literature to predict performance on a number of different criterion tasks such as lexical decision (Connor, Balota, \& Neely, 1992), stem completion (Lupker, Harbluk, \& Patrick, 1991), perceptual identification (Nelson, Gerler, \& Narens, 1984), and of course recognition, to name just a few. Although a complete review of the theories underlying FOKs is beyond the scope of this article, generally speaking, the amount of partial information retrieved determines how strong the FOK rating will be (Koriat, 1993). FOKs are believed to be made heuristically and without a great deal of cognitive effort (see Schwartz, 1994, for a review). This heuristic depiction of FOK judgments is often made because in most experiments the judgments are made only in the absence of recall (but for a different paradigm see Reder, 1987). Just because an item 
cannot be recalled does not necessarily indicate that the rememberer has lost all access to recollective information. Some portion of the partial information retrieved when recall fails could include recollective information, and quite possibly the rememberer might be able to identify these details as supporting later recollection (see also Hicks, Marsh, \& Ritschel, in press).

To extend this reasoning further, FOK judgments based on retrieval of partial information could accurately specify not just whether an item will be recognized but also the rememberer's associated state of awareness accompanying that recognition. In the remember-know literature, recognition judgments are followed by a decision about whether the rememberer has a true state of conscious recollection versus a more undifferentiated feeling of familiarity that arises in the absence of recollection (e.g., Gardiner, Gawlik, \& Richardson-Klavehn, 1994; Rajaram, 1996, 1998; Tulving, 1985). TOTs reflect accurate information about the content of semantic memory, whereas FOKs are believed to reflect accurate information of specific associations formed at encoding. Consequently, FOKs might be predictive of the particular type of awareness that will accompany recognition. The question we are addressing in this study is whether FOK judgments are predictively accurate of subjective states of awareness that will accompany future recognition. In other words, when cued recall fails, will FOKs accurately reflect later remember-know judgments?

To our knowledge, no one has asked this question of the remember-know paradigm. Heuristic theories of how FOKs are made are not consistent, in spirit anyway, with the notion that people would be able to predict that an unrecalled item would be recognized and the associated contextual details would support making a remember judgment. For example, Reder and her colleagues have argued that FOK judgments are based on cue familiarity from a fast preretrieval assessment of the information available in memory (Miner \& Reder, 1994; Reder \& Ritter, 1992). By definition, this theory argues that there is no retrieval of recollective details that supports FOKs. By the same token, Metcalfe's (e.g., 1990) CHARM model replaces the assessment of cue familiarity with an assessment of cue novelty as a basis of FOKs. Both of these theories would predict that FOKs should not be predictive of later recognition remember-know judgments and especially not remember judgments. By contrast, Koriat's (e.g., 1993, 1995) accessibility account argues that FOKs are based on weightings from all information retrieved by cue presentation and not simply made on the basis of undifferentiated familiarity. Because Koriat has made the strong claim that the information that is retrieved could be either correct or incorrect, it is unclear whether the accessibility account predicts that FOKs will be predictive of later remember-know judgments. However, if they are predictive, then accessibility theory would make this prediction much more naturally than those theories of FOKs that are based on simple cue familiarity or novelty. Therefore, the absence of a predictive relationship between FOKs and later remember-know judgments would support heuristic theories of how FOKs are made, whereas evidence of their significant predictive relationship would more likely favor Koriat's accessibility account.

Therefore, we conducted two experiments to determine whether people can use partial information to predict their subjective state of awareness for recognition of unrecalled items. In both experiments, participants studied cue-target word pairs. In the first experiment, participants made FOK judgments to targets that they could not recall in the presence of the cue word at test. Participants then answered a four-alternative forced-choice recognition test that was followed by a remember-know-guess judgment for each unrecallable target. In the second experiment, predictive remember-know-guess judgments replaced the FOK judgments for targets that could not be recalled. In this way, the predictive remember-know judgments could be directly mapped onto participants' reported states of awareness to their recognition judgments. ${ }^{1}$

\section{EXPERIMENT 1}

The purpose of Experiment 1 was to determine whether the partial knowledge that was retrieved in the absence of successful cued recall would be predictive of the state of awareness associated with a subsequent four-alternative recognition judgment. When cued recall was successful, a remember-know-guess judgment assessed the participant's state of awareness for that recollection (see Tulving, 1985, who used a similar procedure). When cued recall failed, participants made a FOK judgment about whether they would recognize the target. After a recognition judgment was made, their state of awareness was assessed using a remember-know-guess judgment and this was then related (i.e., correlated) with their FOK judgment. As is the standard practice in the literature on metacognitive judgments, we used Goodman-Kruskal gamma correlations in assessing this relationship (Nelson, 1984).

\section{Method}

Participants. Thirty-two students from the University of Georgia volunteered in exchange for partial credit toward a course requirement. Each participant was tested individually in sessions that lasted approximately $25 \mathrm{~min}$.

Materials and Procedure. Eighty medium-frequency concrete nouns were used to randomly create 40 cue-target word pairs (e.g., garage-camera). Therefore, these word pairs represented novel associations for the participants. During learning, each word pair was presented in the center of a computer monitor for $5 \mathrm{sec}$ each. The participants were informed prior to study that their memories would be tested on the target member of the cue-target word pair. Word pairs were randomly created anew for each participant.

The participants read the test instructions directly off the computer monitor, and then these instructions were reiterated and clarified by the experimenter. The instructions described the cued recall task, in which the participants were asked to type in the target to each cue or to press the return key if they could not recall the target member of the word pair. If they successfully recalled the target, they 
were asked to make a three-alternative remember-know-guess judgment concerning their subjective state of awareness concerning this recall (see Gardiner, Java, \& Richardson-Klavehn, 1996, for the rationale behind adding a guess category). They had been instructed that when they could not recall the target, they should make an immediate FOK judgment on a 6-point scale including the points $0 \%$, $20 \%, 40 \%, 60 \%, 80 \%$, and $100 \%$ sure that they would recognize the target on a four-alternative forced-choice recognition test that immediately followed the FOK judgment. If the participants made an incorrect recall, the computer made a distinctive error sound and the FOK rating screen appeared. Therefore, the trial sequence was the same for incorrect recalls and admitted recall failures.

After the FOK judgment, the four-item recognition test question appeared. After the participants had chosen a recognition response option, they were instructed to make a remember-know-guess judgment concerning this recognition. The three distractor items for the recognition judgment were drawn from a pool of nonstudied items. We chose distractors in this manner because two-process theories of recognition postulate a mechanism by which an item can be directly recalled and another process by which a decision can be rendered on the basis of an item's familiarity (e.g., Jacoby \& Dallas, 1981; Mandler, 1980). If we had used distractors that were drawn from the study sequence, then the entire cue-target associative structure would have been required for recognition (akin to associative recognition judgments) and that would almost guarantee that only recollection would support recognition. Because our interest was in both remembering and knowing, unrelated distractors were used.

Because it took some time to explain the procedures concerning the FOK and remember-know-guess judgments, there was no explicit retention interval during which the participants performed a distractor activity. After the experimenter was convinced that the participants knew how to make both FOK and remember-knowguess judgments, it took on average approximately $8-10$ min before the test phase was initiated following the encoding phase. This delay seemed to be a suitable enough time frame in which to let rememberknow-guess judgments stabilize (see Rajaram, 1993). Each trial on the 40 -item test proceeded in like fashion, and trials differed only as a function of whether the target was recalled or not. For successfully recalled targets, the remember-know-guess judgment reflected recall and no FOK judgment was required. When recall failed, an FOK judgment predicting recognition performance could be directly related to the subjective state of awareness reported for that recognition decision.

\section{Results and Discussion}

The main data of interest concern performance when target recall fails. Consequently, it would be ideal if the

Table 1

Predictive Judgments (PJs; and Standard Errors) of Recognition and Ultimate Successful Recognition in Experiments 1 and 2

\begin{tabular}{lllllll}
\hline & \multicolumn{2}{c}{$\begin{array}{c}\text { Proportion } \\
\text { Assigned }\end{array}$} & & \multicolumn{2}{c}{$\begin{array}{c}\text { Proportion } \\
\text { Recognized }\end{array}$} \\
\cline { 2 - 3 } Prediction & PJ & $S E$ & & PJ & $S E$ & Cell $n$ \\
\hline Experiment & 1 & & & & & \\
$\quad$ Low & .18 & .04 & & .79 & .04 & 20 \\
Medium & .43 & .03 & & .77 & .04 & 32 \\
High & .39 & .04 & & .93 & .02 & 30 \\
Experiment & & & & & \\
Guess & .27 & .05 & & .72 & .04 & 23 \\
Know & .32 & .04 & & .81 & .03 & 30 \\
Remember & .41 & .05 & .93 & .02 & 29 \\
\hline
\end{tabular}

Note-Cell $n$ refers to the number of participants associated with the proportion of recognized cell means. participants failed to recall the target on the majority of trials. This result was obtained. The proportion of trials in which a target was recalled in the presence of the cue was only $.35(S E M=.04)$. Of this proportion, .31 $(S E M=$ $.04)$ were labeled remember and only $.04(S E M=.01)$ were labeled know. No recalled items were labeled as guesses. The conditionalized probability clearly demonstrates that the majority of recalled targets were labeled remember responses $(86 \%)$ rather than know responses (14\%). This outcome is consistent with Tulving's (1985) empirical results that the majority of responses in free and cued recall were labeled remember rather than know.

On the remaining $65 \%$ of the test trials, the participants either could not recall the target or were incorrect in their recall. In these cases, the participants made an FOK judgment followed by a recognition test decision for the target item that was intermingled among three distractors (randomly chosen anew for each participant). Overall recognition to the $65 \%$ of the targets that went unrecalled was quite good, averaging a proportion correct of .84 (SEM = $.02)$. Thus, the majority of the targets were recognized from the three competing alternatives. Following recognition, the participants made a remember-know-guess judgment. For purposes of presentation we have arbitrarily compressed the 6-point FOK rating scale into 3 points, which should not affect performance (Hays, 1994). These represent low (0\% and 20\%), medium (40\% and 60\%), and high $(80 \%$ and $100 \%)$ confidence in future recognition. The results are summarized in the upper half of Table 1. Because not every participant used every point in the rating scale, we have provided the number of participants contributing to each row of the table in the last column. ${ }^{2}$

The rows of Table 1 correspond to the levels of predictive confidence expressed prior to recognition. The column labeled "proportion assigned" sums to 1.0 and reflects the distribution of predictive confidence that the participants used. As is evident, the participants chiefly expressed more medium and high confidence FOKs than they did low $\left[F(2,62)=7.6, M S_{\mathrm{e}}=.07\right]$. The "proportion recognized" column displays the proportion of targets at each level of confidence that were correctly identified in the four-alternative recognition test. Predictions of low to medium confidence resulted in equivalent recognition performance of approximately $80 \%$, whereas high confidence predictions resulted in over $90 \%$ accurate recognition $\left[F(2,34)=7.8, M S_{\mathrm{e}}=.02\right]$. In general, these results suggest that the participants were somewhat underconfident in their ability to recognize the targets, a finding that is common in the FOK literature (e.g., Hart, 1965). As mentioned earlier, we computed the average GoodmanKruskal gamma correlations between the three levels of confidence and ultimate success (or failure) at recognition. Therefore, this gamma correlation comes from frequency counts in a 3 (confidence) $\times 2$ (recognition) contingency table calculated for each participant tested and then averaged across participants. The mean gamma was .42 (SEM = .11 ), which is significantly greater than a correlation of zero [one sample $t(27)=3.7$ ]. Thus, although participants 
had predictive accuracy for their subsequent recognition performance, it was not perfect accuracy (see also Thiede $\&$ Dunlosky, 1994, for a similar result with recognition and judgments of learning).

The upper half of Table 2 summarizes the data concerning whether the participants' FOK judgments were diagnostic of their ultimate subjective state of awareness on the subsequent recognition test. The data could have been summarized in a number of different ways, but we chose to represent the participants' predictions as columns and their ultimate subjective state of awareness as rows. The data are conditionalizedon their predictions in such a way that each of the three columns for the upper half of Table 2 sums to unity. These judgments in Table 2 represent only the $84 \%$ of the items that were correctly recognized. ${ }^{3}$ When the participants assigned a low probability of recognizing the target, their remember-know judgments were very evenly divided among the three response alternatives of remember, know, and guess $\left[F(2,38)<1.0, M S_{\mathrm{e}}=.15\right]$. These data suggest that items can be subsequently recognized and even labeled either remember or know over $60 \%$ of the time, despite low predictive confidence in even recognizing the target. When the participants had a medium level of predictive confidence, the overwhelming majority of the items were later associated with being known or explicitly remembered $\left[F(2,60)=16.3, M S_{\mathrm{e}}=.08\right]$, with the latter state of awareness representing over $50 \%$ of the recognition decisions. When the participants had high predictive confidence regarding subsequent recognition, the vast majority of these items received a remember judgment $\left[F(2,58)=39.7, M S_{\mathrm{e}}=.07\right]$. The average gamma correlation across participants' 3 (confidence) $\times 3$ (state of awareness) frequency tables was .35 (SEM $=.08)$, which was significantly different from zero by a one-sample test $[t(28)=4.4]$.

Table 2

Predictive Judgments (PJ; and Standard Errors) of Recognition and the State of Awareness Associated With the Recognition Judgment in Experiments 1 and 2

\begin{tabular}{|c|c|c|c|c|c|c|}
\hline \multirow[b]{3}{*}{ Response } & \multicolumn{6}{|c|}{ Prediction } \\
\hline & \multicolumn{2}{|c|}{ Low } & \multicolumn{2}{|c|}{ Medium } & \multicolumn{2}{|c|}{ High } \\
\hline & $\mathrm{PJ}$ & $S E$ & $\mathrm{PJ}$ & $S E$ & $\mathrm{PJ}$ & $S E$ \\
\hline \multicolumn{7}{|l|}{$\begin{array}{l}\text { Experiment } 1 \\
\end{array}$} \\
\hline Guess & .37 & .07 & .12 & .02 & .06 & .01 \\
\hline Know & .31 & .07 & .35 & .05 & .27 & .05 \\
\hline Remember & .31 & .07 & .53 & .05 & .67 & .05 \\
\hline \multirow[t]{4}{*}{$n$} & 20 & & 31 & & 30 & \\
\hline & \multicolumn{6}{|c|}{ Prediction } \\
\hline & \multicolumn{2}{|c|}{ Remember } & \multicolumn{2}{|c|}{ Know } & \multicolumn{2}{|c|}{ Guess } \\
\hline & $\mathrm{PJ}$ & $\overline{S E}$ & $\mathrm{PJ}$ & $\overline{S E}$ & $\overline{\mathrm{PJ}}$ & $\overline{S E}$ \\
\hline
\end{tabular}

Experiment 2

\begin{tabular}{lllllll} 
Guess & .23 & .04 & .18 & .04 & .03 & .01 \\
Know & .28 & .05 & .37 & .06 & .09 & .02 \\
Remember & .49 & .06 & .45 & .06 & .88 & .02 \\
$n$ & 23 & & 30 & & 29 & \\
\hline
\end{tabular}

Note $-n$ gives the total cell count for each column for each experiment.
There was a significant relationship between FOK judgments and the participants' ultimate state of awareness. This relationship appears to be driven largely by medium and high confidence FOKs being associated greater numbers of know and especially remember judgments. Therefore, these data suggest that FOK judgments are predictive of later recognition, which is unsurprising, but are also predictive of later state of awareness associated with recognition, which is the novel finding from this study. The important point is that partial information about the target, perhaps in combination with cue information, is predictive of both future recognition and the future state of awareness about that recognition judgment. One reason that FOKs might be diagnostic of later states of awareness is that a portion of the information that is used to make an FOK judgment is used when participants make rememberknow judgments (or vice versa). That predictive information obviously must contain access to some recollective, contextual details, and this must be true especially for high-confidence FOKs. The implication of these data is that FOK judgments might be less heuristic than is characterized by cue familiarity (Miner \& Reder, 1994) or novelty (Metcalfe, 1990) accounts. Instead, the cognitive process resulting in FOKs appears to take advantage of access to explicit recollective details, as is allowed for in the accessibility account (e.g., Koriat, 1993, 1995). To assess this possibility directly, we had the participants in Experiment 2 make predictive remember-know judgments and we determined whether these judgments would accurately predict remember-know responses that were taken subsequent to recognition.

\section{EXPERIMENT 2}

The results of Experiment 1 suggest that the partial knowledge used to make an FOK judgment is predictive of the reported state of awareness accompanying recognition. The purpose of Experiment 2 was to determine whether participants could use the partial knowledge that was retrieved in the absence of successful cued recall to judge directly their future state of awareness associated with a four-alternative recognition judgment. In this experiment, the participants made predictive rememberknow judgments for items that they could not recall. A recognition test ensued for these unrecallable items and a remember-know judgment was made subsequent to the recognition decision. If the FOK judgments made in Experiment 1 are based on partial recollective information, then predictive remember-know-guess judgments should be diagnostic of future states of awareness. That is, if recollective details are part of what predictive judgments are based on, then those recollective details might be predictive of a future state of remembering after a recognition judgment. By the same token, if that predictive information is vaguer, then perhaps that would be diagnostic evidence of later knowing (as opposed to remembering) subsequent to the recognition judgment. To be clear, we are not claiming that the same information serves as the basis 
of a predictive judgment as the information that is used to render a remember-know-guess recognition judgment. Obviously, postrecognition judgments are influenced by what is retrieved in the presence of the chosen alternative on the recognition test. As such, only a portion of the total information available for making recognition state of awareness judgments would be available during prediction. Therefore, the purpose of the next experiment was to ascertain whether the information available at the time a predictive judgment was made was indicative of later state of awareness.

\section{Method}

Thirty-one participants who had not volunteered in Experiment 1 were recruited. The procedural details were virtually identical to those used before with the exception of replacing the FOK judgment with a predictive remember-know-guess judgment. After the participants had read the instructions, the experimenter reiterated them. The predictive remember-know judgment was described simply to the participants as what they thought they would believe to be true later about remembering, knowing, or guessing after picking the target from among three distractors. Therefore, the predictive judgment had three alternatives, as did the postrecognition remember-knowguess judgment.

\section{Results and Discussion}

For the cued recall task, performance was only $2 \%$ better than in Experiment 1 and therefore basically replicated that experiment. The proportion of targets that the participants recalled was $.37(S E M=.03)$ with $.33(S E M=.03)$ being labeled remember and only .04 $(S E M=.01)$ being labeled know. No recalled target was ever labeled a guess. When conditionalized, these data demonstrate that the overwhelming proportion of the recalled targets were labeled remember (.89), whereas the remaining items (.11) were labeled know. These data are consistent with Experiment 1 and Tulving's (1985) empirical results.

A predictive remember-know judgment was made for the $63 \%$ of the cues on which the target was not recalled. For these predictions, the proportion assigned to each response category is summarized in the first column of data in the lower half of Table 1 in a manner similar to that used in Experiment 1 (recall that entries in columns sum to unity). The predictive assignment to remember, know, and guess categories was fairly equivalent but did exhibit a small nonsignificant trend to be fewer in the guess category than in the know, and more in the remember category than in the know $\left[F(2,60)=1.51, M S_{\mathrm{e}}=.11\right.$, n.s.]. Overall, $83 \%$ of the time the correct alternative was chosen on the recognition test question (and this level of performance is comparable to the $84 \%$ found in Experiment 1 ). The proportion of correct recognition decisions (proportion recognized in Table 1) rose sharply from the guess, through the know, to the remember prediction $[F(2,38)=$ $\left.6.2, M S_{\mathrm{e}}=.03\right]$. As in Experiment 1, these data suggest that the participants were underconfident or conservative in their predictive judgments because the vast majority of the items were subsequently recognized over $70 \%$ of the time even when the participants predicted that they would have to guess at the correct alternative. The gamma correlation between these predictive remember-know judgments and ultimate recognition accuracy was .25 (SEM = .12 ) and that association does differ from a correlation of zero by a one-sample test $[t(28)=2.1]$.

The relationship between the predicted state of awareness and the reported state of awareness for recognition is displayed in the lower half of Table 2. Once again, the data have been conditionalized on predictions with each of the columns summing to unity. When the participants predicted that they would have to guess, the majority of their reported states of awareness were remember responses with equivalent proportions of judgments assigned to the guess and know response categories $[F(2,44)=4.4$, $\left.M S_{\mathrm{e}}=.09\right]$. These data are reassuring in the sense that the participants do not seem to be matching their reported state of awareness to their earlier predictions because average proportions on the upper-left to lower-right diagonal are not the largest proportions in any given column except for remember predictions (i.e., people were willing to change the reported state of awareness from their predicted state). This point is important because it suggests that the information being used to make a predictive judgment is different from the information being used to assess their state of awareness concerning the recognition judgment.

When the participants predicted that they might only know the correct target, most of the items were subsequently given either a know or a remember judgment $\left[F(2,58)=4.6, M S_{\mathrm{e}}=.13\right]$. The fact that postrecognition know responses rise from predictions of guess to know, whereas remember responses do not change, suggests that participants do have access to undifferentiated features that later constitute knowing. That result is not terribly surprising given the cue familiarity and novelty theories that depict predictions being made heuristically. By contrast, participants quite accurately predicted that remembered items would be remembered when given the recognition test, with very few of these items being given guess or know responses $\left[F(2,56)=424.8, M S_{\mathrm{e}}=.02\right]$. Therefore, although the participants may have some predictive access to undifferentiated features, this last result suggests that they have better predictive access to recollective details in the partial information retrieved in their search for the targets. The interesting aspect of these data is that they gave rise to the largest gamma correlation found across these two experiments. The correlation was .56 (SEM = $.07)$, which is significantly greater than zero by one-sample test $[t(29)=7.6]$.

The results of Experiment 2 were similar to those found in Experiment 1. In general, both FOK judgments and predictive remember-know judgments show that participants are underconfident in their predictions. One striking aspect of this experiment was that the participants predicted that $41 \%$ of their judgments would be remember judgments (Table 1), and ultimately they did recognize the correct alternative $93 \%$ of the time on these trials and subsequently gave it a postrecognition remember judgment 
$88 \%$ of the time. Although the participants could have been matching their retrospective remember-know-guess judgment to their prediction, the data from the guess and know predictions strongly argue that they are basing these judgments on different sorts of information that are available at different points in the experimental sequence. Therefore, these data suggest that people do have access to partial information and that some of that information contains sufficient recollective details about the unrecalled target to successfully predict that it will be remembered.

\section{GENERAL DISCUSSION}

Our goal was to assess whether the partial knowledge available when an item is not accessible to cued recall processes is nevertheless predictive of one's future state of awareness during a recognition process. The answer to that question is a strong affirmative one, with the small qualification that it may depend slightly on the type of prediction being made. Traditionally, only TOT states, FOK judgments, and speeded strategy decisions (e.g., Reder, 1987; Reder \& Ritter, 1992) have been used to assess whether partial knowledge in the absence of retrieval is correlated with ultimate performance. We introduced a predictive remember-know-guess judgment in Experiment 2 to address our question of whether people have access to undifferentiated features that give rise to knowing as well as more recollective information that supports remembering without the explicit ability to recall target items. They do, with the novel finding that people can predict remember responses with moderate accuracy, and therefore, must have some partial access to recollective details in memory.

We have characterized the nature of a memory trace as bundles of different features that are established at encoding. We predicted that some of these features would be among the partial information retrieved when cued recall failed, and further, that some of these features would contain recollective information. Obviously, some of these features are recollective in nature because they support predictive remember judgments in Experiment 2. Traditional cognitive theories in which judgments can be made either heuristically or more systematically do not usually associate heuristic cognitive processes with the inspection of recollective details (e.g., Johnson et al., 1993). Arguably, it would be something of an oxymoron to do so. Therefore, the data from both experiments suggest that highconfidence FOK judgments and predictive rememberknow-guess judgments are probably informed by, and can make good use of, recollective details in a more systematic way than has been portrayed previously in the FOK literature except by Koriat's accessibility account (e.g., Koriat, 1995). For example, Reder and Ritter (1992) have strongly emphasized that the FOK process is an automatic preretrieval process and the retrieval process itself is an effortful, controlled process. Although their argument may apply specifically to their speeded judgment paradigm, our data argue otherwise in a more general way for more typical FOK experiments using paired associates or TOT states. FOKs and similar predictive judgments can use the partially retrieved products of attempted (but failed) recall and can do so to specify that there are sufficient recollective details present in memory that something to be recognized later will be labeled remembered rather than merely known. Thus, the data do seem to qualify in an absolute sense the stance that FOK judgments are always made heuristically (see Schwartz, 1994, for a summary of such claims). However, as we stated earlier, we are not saying that the same information that is being used to predict performance is being used to make rememberknow judgments subsequent to recognition.

Of course, we have used novel associations and participants were informed that their memory would be tested. Perhaps this led our participants to use mnemonic strategies that left a great deal of recollective information that might not otherwise be available outside laboratory settings or in different laboratory experiments. For example, if category-exemplar word pairs (e.g., fruit-apple) had been used instead and only the category label was provided as a cue at test, then there would likely be less recollective information available because all of the associations tested would be preexisting semantic associations as would the distractor items on the recognition test (e.g., other fruits). Perhaps under these circumstances the predictive remember-know judgments would not be as accurate as was found in Experiment 2, or perhaps predictions of know responses would outweigh remember responses. However, even if such predictions came to pass, such results would not contravene the fact that people can take into account partial recollective information (if available) when making predictions about their future memory performance as they obviously did in Experiment 2. In sum, the present results are clear that the information available when recall fails is definitely diagnostic of people's future state of awareness for recognizing an unrecallable item.

\section{REFERENCES}

ANISFIELD, M., \& KNAPP, M. (1968). Association, synonymity, and directionality in false recognition. Journal of Experimental Psychology, 77, 171-179.

Bower, G. H. (1967). A multi-component theory of the memory trace. In K. W. Spence \& J. T. Spence (Eds.), The psychology of learning and motivation (Vol. 1, pp. 229-325). New York: Academic Press.

Brown, R., \& McNeIll, D. (1966). The "tip of the tongue" phenomenon. Journal of Verbal Learning \& Verbal Behavior, 5, 325-337.

Connor, L. T., Balota, D. A., \& Neely, J. H. (1992). On the relation between feeling of knowing and lexical decision: Persistent subthreshold activation or topic familiarity? Journal of Experimental Psychology: Learning, Memory, \& Cognition, 18, 544-554.

Dewhurst, S. A., \& Conway, M. A. (1994). Pictures, images, and recollective experience. Journal of Experimental Psychology: Learning, Memory, \& Cognition, 20, 1088-1098.

Gardiner, J. M., Gawlik, B., \& Richardson-Klavehn, A. (1994). Maintenance rehearsal affects knowing, but not remembering; elaborative rehearsal affects remembering, not knowing. Psychonomic Bulletin \& Review, 1, 107-110.

Gardiner, J. M., JaVa, R. I., \& Richardson-Klavehn, A. (1996). How level of processing really influences awareness in recognition memory. Canadian Journal of Experimental Psychology, 50, 114-122.

Hart, J. T. (1965). Memory and the feeling-of-knowing experience. Journal of Educational Psychology, 56, 208-216. 
HaYs, W. L. (1994). Statistics. New York: Harcourt Brace.

Hicks, J. L., Marsh, R. L., \& Ritschel, L. (in press). The role of recollection and partial information in source monitoring. Journal of Experimental Psychology: Learning, Memory, \& Cognition.

JACOBY, L. L., \& DALlas, M. (1981). On the relationship between autobiographical memory and perceptual learning. Journal of Experimental Psychology: General, 110, 306-340.

Johnson, M. K., Hashtroudi, S., \& Lindsay, D. S. (1993). Source monitoring. Psychological Bulletin, 114, 3-28.

KorIAT, A (1993). How do we know what we know? The accessibility model of the feeling of knowing. Psychological Review, 100, 609-639.

KorIat, A. (1995). Dissociating knowing and the feeling of knowing: Further evidence for the accessibility model. Journal of Experimental Psychology: General, 124, 311-333.

Koriat, A., \& LIEBLICH, I. (1974). What does a person in a "TOT" know that a person in a "don't know" state doesn't know? Memory \& Cog nition, 2, 647-655.

Lupker, S. J., Harbluk, J. J., \& Patrick, A. S. (1991). Memory for things forgotten. Journal of Experimental Psychology: Learning, Memory, \& Cognition, 17, 897-907.

MANDLER, G. (1980). Recognizing: The judgment of previous occurrence. Psychological Review, 87, 252-271.

Metcalfe, J. (1990). Composite holographic associative recall model (CHARM) and blended memories in eyewitness testimony. Journal of Experimental Psychology: General, 119, 145-160.

Metcalfe, J., Schwartz, B. L., \& Joaquim, S. G. (1993). The cue familiarity heuristic in metacognition. Journal of Experimental Psychology: Learning, Memory, \& Cognition, 19, 851-861.

Miner, A. C., \& Reder, L. M. (1994). A new look at feeling of knowing: Its metacognitive role in regulating question answering. In J. Metcalfe \& A. P. Shimamura (Eds.), Metacognition: Knowing about knowing (pp. 47-70). Cambridge, MA: MIT Press.

Nelson, T. O. (1984). A comparison of current measures of the accuracy of feeling-of-knowing predictions. Psychological Bulletin, 95, 109-133.

Nelson, T. O., Gerler, D., \& Narens, L. (1984). Accuracy of feeling of knowing judgments for predicting perceptual identification and relearning. Journal of Experimental Psychology: General, 113, 282-300.

RAJARAM, S. (1993). Remembering and knowing: Two means of access to the personal past. Memory \& Cognition, 21, 89-102.

Rajaram, S. (1996). Perceptual effects of remembering: Recollective processes in picture recognition memory. Journal of Experimental Psychology: Learning, Memory, \& Cognition, 22, 365-377.

RAJARAM, S. (1998). The effects of conceptual salience and perceptual distinctiveness on conscious recollection. Psychonomic Bulletin \& Review, 5, 71-78.

Reder, L. M. (1987). Selection strategies in answering questions. Cognitive Psychology, 19, 90-138.

REder, L. M., \& Ritter, F. E. (1992). What determines initial feeling of knowing? Familiarity with question terms, not with the answer.
Journal of Experimental Psychology: Learning, Memory, \& Cognition, 18, 435-451.

SchWARTZ, B. L. (1994). Sources of information in metamemory: Judgments of learning and feelings of knowing. Psychonomic Bulletin \& Review, 1, 357-375.

Thiede, K. W., \& Dunlosky, J. (1994). Delaying students' metacognitive monitoring improves their accuracy in predicting their recognition performance. Journal of Educational Psychology, 86, 290-302.

Tulving, E. (1985). Memory and consciousness. Canadian Psychologist, 40, 1-12.

Tulving, E., \& Pearlstone, Z. (1966). Availability versus accessibility of information in memory for words. Journal of Verbal Learning \& Verbal Behavior, 5, 381-391.

Tulving, E., \& Thomson, D. M. (1973). Encoding specificity and retrieval processes in episodic memory. Psychological Review, 80, 352373.

YANIV, I., \& MeYER, D. E. (1987). Activation and metacognition of inaccessible information: Potential bases for the incubation effect in problem solving. Journal of Experimental Psychology: Learning, Memory, \& Cognition, 13, 187-205.

\section{NOTES}

1. The reader will notice that we have skirted the issue of whether remember-know judgments reflect retrieval of information from distinct memory systems versus being based on a unidimensional continuum of trace "strength" along which different decision criteria are set. Our inquiry was intended neither to resolve nor to fuel this debate. Regardless of the theoretical position one chooses to adopt, this study merely investigated whether the partial knowledge retrieved during unsuccessful cued recall is sufficient to support accurate judgments of people's future state of awareness. Whether people retrieve from separate memorial systems or base their judgments only on the amount of familiarity is immaterial for the present purposes.

2. For the same reason, the degrees of freedom reported for the statistical tests will vary as function of the number of participants contributing to all cells of within-subjects comparisons. The means reported in the tables did not change by more than a percentage point or two.

3 . The remaining $16 \%$ were incorrect recognition responses and will not be reported because the data are not sufficient for meaningful analysis. For these incorrect recognition decisions, suffice it to say that regardless of whether the prediction was a low, medium, or high FOK, the vast majority of the items received a guess to characterize the subjective state of awareness associated with recognition. The same was true of the $17 \%$ incorrect recognition decisions reported in Experiment 2 as well.

(Manuscript received November 24, 2000; revision accepted for publication August 28, 2001). 